\title{
EFFECT OF NAOH MERCERISATION ON THE CROSS LINKING OF CONVENTIONAL AND COMPACT COTTON YARNS USING DMDHEU RESIN
}

\author{
Shabiya Thaseen \\ Department of Home Science, Textile Science and Fashion Designing, J.B.A.S. College for Women \\ Teynampet, Chennai - 600018
}

\begin{abstract}
An investigation of the effect of resin finishing on the properties of conventional and compact cotton yarn is reported. It is shown that compact yarns suffer greater weight losses than those of conventional yarns following cross linking treatment. Bending rigidity shows an increase with the concentration of sodium hydroxide prior to cross linking in both conventional and compact yarns. The yarn structure also plays an important role in resin finishing.
\end{abstract}

\section{INTRODUCTION}

Durable press finishing processes are used in the textile industry to produce wrinkle resistant cotton fabrics and garments. The finishing agents used are N-methylol reagents such as dimethylol dihydroxy ethylene urea (DMDHEU) which were conventional durable press finishing agents. Recently, the use of polycarboxylic acids such as $1,2,3,4$ butane-tetra carboxylic acids (BTCA) and citric acid, tartaric acid, maleic acid and itaconic acid due to increasing concern with toxicity and the adverse impact on the environment by formaldehyde has become widespread $(1,2,3,4)$.

A durable press finishing agent forms covalent bonds with cellulosic hydroxyl groups under elevated temperatures, thus forming cross linkages between the cellulose molecules. Cross linking normally leads to an improvement in wrinkle resistance and reduces the strength of cotton fabric. Wrinkle recovery and tensile strength are the two principal parameters which are used to assess the performance of the cross linked cotton fabrics.

Yang et al (5) have conducted detailed research on the nonformaldehyde finishes and their results are quite useful. Formation of anhydroxide with sodium hypophosphite as a catalyst and chemical analysis of 1, 2, 3, 4 - Butane tetra carboxylic acid using FTIR and FT-Raman Spectroscopy, proton magnetic resonance spectroscopy (1H-NMR) mass spectroscopy (MS) and liquid chromatography mass spectroscopy LC/MS have been carried out. All the instrumental analysis data indicate the low level of impurities in the industrial BTCA Yang is credited with a number of papers on the analysis of BTCA treated fabrics.
In this paper, the effect of cross linking on the performance of conventional and compact cotton yarns is discussed using DMDHEU. The yarn characteristics namely, yarn strength, elongation and bending were measured for the treated conventional and compact yarns. This work provides the basic data to know and understand the role of anticreasing yarns in the fabric. Although it is rather unusual to apply resin finishes to yarns, nevertheless in order to gain some basic knowledge this work was carried out. Moreover, it is of interest to know the response of yarn structure to resin treatments.

\section{EXPERIMENTAL}

\subsection{Materials}

Yarns of $30 \mathrm{Ne}$ produced by conventional and compact spinning technologies from the same cotton mixing were used. They were bleached. DMDHEU and catalyst were used. The prepared yarns in hank form were immersed in DMDHEU formulation for 10 minutes. Triton X-100, 1\% PEG 400 and $1.8 \%$ magnesium chloride hexahydrate.

DMDHEU is the most commonly used crosslinking agent and contains urea, formaldehyde and glyoxal. The two methylol groups and the two hydroxyl groups of DMDHEU react with cellulosic hydroxyl to form cross linkages between cellulose molecules. The yarns were treated with DMDHEU solution for 10 minutes at concentrations namely 80, 100, 120 and 130 gpl. The wet pick up of the impregnated yarns was in the range of $100-105 \%$. The yarns following impregnation were dried at $100^{\circ} \mathrm{C}$ in a forced air oven and cured at $160^{\circ} \mathrm{C}$ for 3 min, and washed with $5 \mathrm{gpl} \mathrm{Na} 2 \mathrm{CO} 3$ at $50^{\circ} \mathrm{C}$ for 5 minutes to remove the untreated chemicals then rinsed to remove the reagent not bound to the cotton yarns and dried. 
A portion of conventional and compact yarns was treated with sodium hydroxide at various concentrations and subsequently cross linked with DMDHEU. This was done to find out the effect of concentration of $\mathrm{NaOH}$ on the efficacy of cross linking. In the earlier case, they were treated with $20 \% \mathrm{NaOH}$. The cured yarns were evaluated for strength and elongation using Uster Tensorapid tester. They were also tested for bending rigidity.

\subsection{Bending Rigidity}

Bending rigidity was measured by using Ring-loop method developed by Carlene (7). In this method, a length of yarn of filament is bent through $360^{\circ}$ to form a ring. The ends are gripped and the distortion produced in the ring shape by some externally applied weight is observed. In these conditions, the flexural rigidity $\mathrm{G}$ is given by the equation.

$$
\mathrm{G}=\mathrm{KWL}^{2} \frac{\cos \theta}{\tan \theta}
$$

where,

$\mathrm{K}=\mathrm{a}$ constant the value of which is around 0.0047

$\mathrm{W}=$ applied load in $\mathrm{mg}$

$\mathrm{L}=$ Circumferential length of undistorted ring in $\mathrm{cm}$

$$
\theta=\frac{493 \mathrm{~d}}{\mathrm{~L}}
$$

$\mathrm{d}=$ deflection of lower end of the ring under action of applied load. For grater sensitivity, a value of $\mathrm{W}$ is chosen that $\theta$ lies between $40^{\circ}$ and $50^{\circ}$. When $\mathrm{W}$ is given in grams and $\mathrm{L}$ and $\mathrm{d}$ in centimetres, the flexural rigidity is expressed in g.cm2. For each sample, ten tests were done and the average was taken. Full details are given in the thesis (6).

\section{RESULTS AND DISCUSSION}

The results included in Table 1 show that the strength losses following resin finish are greater $(28 \%)$ for compact yarns in comparison with conventional yarns $(10 \%)$ elongation shows an increase with increase in resin content.

Table 2 gives the results of tenacity and elongation of conventional and ompact yarns mercerised in slack form at different concentrations. Elongation shows an increase with increase in the concentration of sodium hydroxide. This is due to shrinkage of yarns following mercerisation.

Tensile properties of conventional and compact yarns which were slack mercerised at different concentrations and cross linked are shown in Table 3. There is a significant drop in strength of cross linked compact yarn in comparison with conventional yarns. Elongation shows an increase in both the yarns.

Cross linking of cotton cellulose molecules causes fibre embrittlement which results in loss in strength.

Table 1 Tensile properties of conventional and compact yarns cross linked by DMDHEU

\begin{tabular}{|l|l|l|l|l|}
\hline & \multicolumn{2}{|l|}{ Conventional } & Compact \\
\cline { 2 - 5 } & $\begin{array}{l}\text { Tenacity } \\
(\mathrm{cN} / \text { tex })\end{array}$ & $\begin{array}{l}\text { Elongation } \\
\%\end{array}$ & $\begin{array}{l}\text { Tenacity } \\
(\mathrm{cN} / \text { tex })\end{array}$ & $\begin{array}{l}\text { Elongation } \\
\%\end{array}$ \\
\hline Control & 17.21 & 4.66 & 18.49 & 5.14 \\
\hline $80 \mathrm{GPL}$ & 14.86 & 6.65 & 15.05 & 6.64 \\
\hline $100 \mathrm{GPL}$ & 16.22 & 7.06 & 14.72 & 6.21 \\
\hline $120 \mathrm{GPL}$ & 14.8 & 6.51 & 14.56 & 6.47 \\
\hline $130 \mathrm{GPL}$ & 15.47 & 6.62 & 14.98 & 6.35 \\
\hline
\end{tabular}

Table 2 Effect of concentration of alkali on the conventional and compact yarn characteristics

\begin{tabular}{|l|l|l|l|l|}
\hline \multirow{2}{*}{ Conc. of $\mathrm{NaOH} \%$} & \multicolumn{2}{|l|}{ Conventional } & \multicolumn{2}{l|}{ Compact } \\
\cline { 2 - 5 } & $\begin{array}{l}\text { Tenacity } \\
(\mathrm{cN} / \text { tex })\end{array}$ & $\begin{array}{l}\text { Elongation } \\
\%\end{array}$ & $\begin{array}{l}\text { Tenacity } \\
(\mathrm{cN} / \text { tex })\end{array}$ & $\begin{array}{l}\text { Elongation } \\
\%\end{array}$ \\
\hline 0 & 17.21 & 4.66 & 18.49 & 5.14 \\
\hline 4 & 17.33 & 5.12 & 18.52 & 5.42 \\
\hline
\end{tabular}




\begin{tabular}{|c|c|c|c|c|}
\hline 8 & 17.86 & 5.41 & 18.62 & 5.48 \\
\hline 12 & 17.92 & 5.45 & 18.54 & 6.21 \\
\hline 14 & 18.21 & 6.32 & 18.32 & 6.34 \\
\hline 16 & 18.32 & 7.4 & 18.28 & 6.45 \\
\hline 18 & 18.56 & 8.6 & 18.41 & 7.48 \\
\hline 20 & 18.96 & 9.9 & 18.56 & 8.92 \\
\hline
\end{tabular}

Table 3 Tensile and bending properties of conventional and compact cotton yarn treated by $\mathrm{NaOH}$ with zero tension and cross linked by DMDHEU

\begin{tabular}{|l|l|l|l|l|l|l|}
\hline \multirow{2}{*}{$\begin{array}{l}\text { Conc. } \\
\text { NaOH \% }\end{array}$} & \multicolumn{2}{|l}{ After cross linking (conventional) } & \multicolumn{2}{l}{$\begin{array}{l}\text { After } \\
\text { (compact) }\end{array}$} \\
\cline { 2 - 7 } & $\begin{array}{l}\text { Tenacity } \\
(\mathrm{cN} / \text { tex })\end{array}$ & $\begin{array}{l}\text { Elongation } \\
\%\end{array}$ & $\begin{array}{l}\text { Bending } \\
\text { rigidity } \\
(\mathrm{mN} . \mathrm{mm} 2)\end{array}$ & $\begin{array}{l}\text { Tenacity } \\
(\mathrm{cN} / \text { tex })\end{array}$ & $\begin{array}{l}\text { Elongation } \\
\%\end{array}$ & $\begin{array}{l}\text { Bending } \\
\text { rigidity } \\
(\mathrm{mN} . \mathrm{mm} 2)\end{array}$ \\
\hline 0 & 17.04 & 4.21 & 1.5 & 18.32 & 4.82 & 1.6 \\
\hline 4 & 16.82 & 5.01 & 2.0 & 17.86 & 4.68 & 2.1 \\
\hline 8 & 16.72 & 4.89 & 2.2 & 17.72 & 4.52 & 2.3 \\
\hline 12 & 16.34 & 5.21 & 2.5 & 16.84 & 4.62 & 2.7 \\
\hline 14 & 15.89 & 5.11 & 2.8 & 17.28 & 4.68 & 2.7 \\
\hline 16 & 15.32 & 5.09 & 3.0 & 17.44 & 4.56 & 3.4 \\
\hline 18 & 18.72 & 5.12 & 3.2 & 17.28 & 4.61 & 3.6 \\
\hline 20 & 19.36 & 8.6 & 3.5 & 16.84 & 4.72 & 3.8 \\
\hline
\end{tabular}

The reason for the drop in strength in the case of compact yarn can be due to greater embrittlement following resin treatment. Since the packing coefficient is higher in compact yarns, following resin treatment the material becomes still more compact which leads to greater strength losses.

\subsection{Bending Rigidity}

It is apparent that flexural rigidity increases with an increase in concentration of sodium hydroxide prior to the resin treatment for conventional and compact yarns. This is due to no freedom of motion of fibres in the resin treated yarns as pointed out by Backer (9). The bending moment is higher in the case of no freedom of motion. Platt (8) also computed the bending moment for the case of no freedom of motion.

\section{CONCLUSIONS}

This study mainly investigates the effect of $\mathrm{NaOH}$ mercerisation on the crosslinking properties of cotton yarns made by conventional and compact spinning technologies. Compact yarns suffer great strength losses than conventional yarns. The results show that mercerisation has a considerable effect on crosslinking properties such as tensile strength and elongation of yarns. Further studies on fabrics made out of these yarns are in progress, and the results will be reported later.

\section{ACKNOWLEDGEMENTS}

The author would like to acknowledge her indebtedness to Dr. Venkatraman Subramaniam, Jaya Engineering College, Chennai for his various advices. 


\section{REFERENCES}

[1] Yang, C.Q., and Bakshi, G.D., Quantitative analysis of the nonformaldehyde durable press finish on cotton fabric - Acid-base titration and infrared spectroscopy, Textile Res. J., 66(6), 1996, 377-384.

[2] Edwin Sunder, E. and Nalankilli, G., Polyfunctional finishes on cotton textiles. Ind. J. Fib and Text Res., 37, 2012, 364-371.

[3] Welch, C.M., Tetracarboxylic acid as formaldehyde free durable press finishing agents, Textile Res. J., 58(8), 1988, 480-486.

[4] Yang, C.Q., Lu, Y. and Lickfield, G.C., Chemical analysis of 1,2,3,4-Butane tetracarboxylic acid, Textile Res J., 72(9), 2002, 817-824.

[5] Yang, C.Q. and Wang, X., “Applications of FT-IR spectroscopy to the studies of esterification and crosslinking of cellulose by polycarboxylic acids, Part II. The performance of the crosslinked cotton fabrics", in Fourier Transform Spectroscopy Eleventh International Conference James A. De Haseth Ed., American Institute of Physics, Woodbury, New York, 1998, pp.665-669.

[6] Shabiya Thaseen, "An investigation of the yarn characteristics of conventional and compact cotton yarns and dyeing behaviour of man made cellulosic fabrics", Ph.D., Thesis, Mother Teresa Women's University, Kodaikanal, 2011.

[7] Carlene, P.W., "The relation between fibre and yarn flexural rigidity in continuous filament viscose yarns", J. Text. Inst., Vol.41, No.5, 1950, T159-T171.

[8] Platt, M.M., Klein, W.G., Hamburger, W.J., Some aspects of bending rigidity of single yarns", Textile Res J., 29, 1959, 611.

[9] Backer, S., "The mechanics of bent yarns", Textile Res. J., 22, 1952, 668. 